$\mathrm{Zn}$-induced sheets, the relative arrangement of tubulin protofilaments appears to be different from that in the 'normal' sheets and in microtubules, but the protofilaments seem to be essentially the same.

The results show the structure in more detail than has been observed before, although the present resolution is still only about $20 \mathrm{~A}$. Some preliminary studies on unstained sheets suggest that it may be possible eventually to achieve the sort of high resolution results obtained by Henderson and Unwin in their study by electron microscopy of the purple membrane from Halobacterium (Nature 257, 28; 1975).

\section{Primate social structure and ecology}

from John Krebs

Gibbons (Hylobates spp.) live in pairs and defend a territory of less than 1 square kilometre; baboons (Papio spp.) wander over an undefended home range of 15-20 square kilometres in polygamous or promiscuous groups of about 40 individuals. These contrasts in group size, home range, mating system and so on are typical of the bewildering array of social organisations in primates, and one of the major aims of primate ethologists over the past 15 years or so has been to seek regular patterns in the differences between species.

J. H. Crook and J. S. Gartlan (Nature 210, 1200; 1966) made the first serious attempt to link primate social structure with a small number of ecological variables such as food dispersion and pressure from predators. They noted, to take just one example, that ground-living primates such as baboons live in larger groups than their arboreal relatives and suggested that this could be an antipredator adaptation, terrestrial monkeys being more exposed to predators and hence benefiting more from communal defence in a large group. Crook and Gartlan clearly had the right approach, but their attempt to classify primate societies into about half a dozen ecological categories foundered because as more field evidence came to hand, too many exceptions to their rules emerged. This led to two modifications of the original approach. Some argued that Crook and Gartlan were wrong to assume that primate social organisation is always adapted to present day conditions, and that phylogenetic heri-

John Krebs is a lecturer in Zoology at the Edward Grey Institute of Field Ornithology, University of Oxford.

\title{
Nitrogen diffusion in diamond
}

from John Walker

THE results on conversion of diamonds from type Ib to type Ia which are reported in this issue of Nature by Chrenko, Tuft and Strong (page 141) have important implications for diamond physics and for synthesis of gemstones.

The Platonically ideal diamond is composed only of carbon. Real diamonds, however, contain impurities, and paradoxically, the large, regularly shaped and apparently perfect gemstones are usually the most impure. (This effect is well-known to crystal-growers-impurities affect the shape of many types of crystal.) In diamond the most important impurity is nitrogen. A diamond is classified as type I if it is appreciably impure, and as type II if it is relatively nitrogen-free. The nitrogen may be present as isolated individual atoms (type $\mathrm{lb}$ ), giving the diamond a yellow colour, a paramagnetic resonance signal and a characteristic infrared absorption; or it may be in aggregates, especially pairs (type Ia), resulting in no paramagnetism, no visible colour and a different infrared spectrum. This type-classification is qualitative, and diamonds of mixed type are often found, but it is useful nonetheless.

The vast majority of natural diamonds are type $\mathrm{Ia}$, but synthetic crystals are usually type Ib. Nobody knows why for sure; a plausible hypothesis is that some natural diamonds at least, started life as type Ib, and were subsequently transformed to the Ia type.

Diamond is the metastable form of carbon at normal temperature and pressure. It is stable, and so can be synthesised, at higher temperature and pressure. The hypothesis is that, in some conditions, although nitrogen can be incorporated into the growing crystal, it is immobile; subsequent higher temperatures or lower pres-

tage plays a strong role (Struhsaker Folia Primat. 11, 80; 1969) while others proposed that Crook and Gartlan were in principle right, but that the differences in ecological pressures had to be analysed on a much finer scale (Clutton-Brock Nature 250, 539; 1974), and further that the same ecological pressure may lead to different solutions.

In an important new review, $T$. $H$. Clutton-Brock and P. H. Harvey J. Zool. Lond. 183, 1; 1977) have greatly extended and updated Crook sures allow it to become mobile and diffuse through the carbon lattice until it can aggregate with other nitrogen atoms. Chrenko et al. report that after annealing at $2,000 \mathrm{~K}$ and 60,000 atmospheres for $30 \mathrm{~min}$ the type Ib diamonds had become appreciably paler yellow in colour, due to reduction of their characteristic visible absorption, and that their paramagnetism was weaker. The infrared spectrum had changed from typical type $\mathrm{Ib}$ to mixed $\mathrm{Ia}$ and $\mathrm{Ib}$, and the N3 ultraviolet absorption system (thought to be an aggregate of three nitrogen atoms) could be detected. The total nitrogen concentration remained constant-a useful consistency check.

What is the significance of these results? First, they tell us more about how nitrogen behaves in diamond; and they may help us to understand other defects, especially the 'platelets'-laminar defects on $\{100\}$ planes which have intrigued and puzzled diamond physicists for 40 years. Second, they indicate a method of turning yellow synthetic diamonds into 'water-white' ones, thus enhancing their value as gemstones. Third, they tell us more about the synthesis process, which may lead to the commercial synthesis of large gem-quality diamonds. (At present, although tons of synthetic diamonds are produced annually, they are suitable only for industrial applications -it is actually cheaper to dig the gemstones out of the ground than it is to synthesise them.) Finally, the results may have implications for the conditions prevailing in the Earth's mantle that led to the natural synthesis of diamonds. As Sir Charles Frank put it, 'Nakaya has written a poem in which he says, "A snowflake is a letter to us from the sky." A diamond is a letter to us from the depths, and a letter more worth reading because we can visit the sky.'

and Gartlan's original approach. They have classified all the primate species for which they could find data into nocturnal or diurnal, arboreal or terrestrial, and insectivorous, frugivorous or folivorous. They then did a quantitative statistical analysis to look for consistent differences between these ecological categories in features such as group size, home range, sex ratio and body weight. The main trends to emerge from their analyses were that nocturnal primates which are all arboreal and mainly eat either 